\title{
Todes - Anzeige.
}

La sera del r 9 Agosto I 886 moriva improvvisamente nella villa di Borgo San Pietro presso Torino il Prof. Alessandro Dorna, direttore dell' Osservatorio Astronomico della R. Università di Torino. Nato il 13 Febbrajo 1825 in Asti, il Dorna fu laureato Ingegnere Idraulico il 5 Agosto 1848 , chiamato ad insegnare Meccanica Razionale nel $185^{\circ}$ all Accademia Militare, e finalmente eletto a succedere al Plana nella direzione della Specola e nella cattedra di Astronomia il i 8 Settembre 1865. Appartenne all' Istituto Lombardo, all' Accademia dei Lincei ed all' Accademia delle Scienze di Torino, alla quale presentò quasi esclusivamente i suoi lavori sopra argomenti di meccanica, di meteorologia e di astronomia. Nel i $874 \mathrm{fu}$ con altri astronomi inviato nell' India, a Muddapur, dove prese parte alle osservazioni del passaggio di Venere sul disco solare. F. Porro.

\section{Entdeckung eines neuen Cometen von Finlay.}

Die Centralstelle für Astr. Telegramme erhielt heute Vormittag folgendes Telegramm:

*Unharmed Finlay unbound September landsman jacobite committible fishy gingle smallness sluggishness unhappy cordage. Astronomer Capetown. *

d. h. Ein Comet wurde von Finlay am 26 . September entdeckt und beobachtet:

Sept. 26.3339 M. Z. Gr. AR. $=255^{\circ} 30^{\prime} 28^{\prime \prime}$, PD. $=116^{\circ} 4^{\prime} 6^{\prime \prime}$. Tägl. Bew. $+35^{\prime}$ in AR., + $4^{\prime}$ in PD. Der Comet ist rund, I Minute im Durchmesser, $\mathbf{I}^{\mathrm{m}}$ oder schwächer, hat einige centrale Verdichtung, ohne Schweif.

Das Telegramm wurde sofort weiter befördert, auch an die Sternwarten der südlichen Halbkugel.

Kiel 1886 Sept. 28 .

A. Krueger.

\section{Vermischte Nachrichten.}

(Aus dem Anzeiger der Wiener Akademie der Wissenschaften 1886 Nr. 16.)

er die Berechnung der Präcession mit besonderer Rücksicht auf die Reduction eines Sterncataloges auf eine andere Epoche, von Director E. Weiss.

Durch eine Entwicklung der Gleichung, welche für einen beliebigen Zeitraum den Betrag der Präcession eines Gestirnes in Rectascension und Declination giebt nach steigenden Potenzen des Sinus des Winkels, den die Aequatoren $z u$ beiden Zeiten $t_{0}$ und $t$ mit einander einschliessen, gelangt der Verfasser für den Betrag der Präcession in der Zwischenzeit zu einem Ausdrucke von der Form:

$\alpha-\alpha_{0}=\lambda+x \sin \left(\alpha_{0}+p\right) \operatorname{tg} \delta_{0}+\mu \sin ^{2}\left(\alpha_{0}+p\right)\left[\mathbf{r}+2 \operatorname{tg}^{2} \delta_{0}\right]+\cdots$ $\delta-\delta_{0}=x \cos \left(\alpha_{0}+p\right)+\mu^{\prime} \sin ^{2}\left(\alpha_{0}+p\right) \operatorname{tg} \delta_{0}+\cdots$

wobei die Grössen $p, x, \lambda, \mu, \mu^{\prime} \ldots$ nur Functionen der
$Z$ wischenzeit $t-t_{0}$, also vom Sternorte unabhängig sind, und wobei, wenn man die Präcession als eine Grösse erster Ordnung auffasst, $\boldsymbol{p}, \boldsymbol{x}$ und $\lambda$ Grössen erster Ordnung, $\mu$ und $\mu^{\prime}$ solche zweiter Ordnung vorstellen und die weiteren Glieder höheren Ordnungen angehören.

Der Verfasser sucht den Schwerpunkt seiner Entwicklung darin, dass die obige Formel sich sehr leicht und bequem tabuliren lässt, und damit die Möglichkeit an die Hand giebt, einen Sterncatalog mit Leichtigkeit von einer Epoche auf die andere zu ibertragen, was nach den bisher üblichen Berechnungsmethoden der Präcession bekanntlich nicht der Fall ist.

Um die leichte Anwendbarkeit des Verfahrens dar. zuthun, werden zum Schlusse die nöthigen Hülfstafeln beigegeben, um Sterne von den Epochen I800.0, I825.0, I 875.0 und 1880.0 auf 1850.0 zu reduciren.

Verkaufs-Anzeige. W. Wesley \& Son, 28 Essex St., Strand, London, offeriren: British Association Catalogue of Stars, 1845 , cloth. M. 170.

\section{Inhalt:}

Zui Nr. 2748.49. Perrotin. Mesures micrométriques d'étoiles doubles, 193. -- F. E. Gore. 'On the Orbit of the Binary Star $\tau$ Cygni. 215. S. C. Chandler, jr. On the Variable ro Sagittae $19^{\mathrm{h}} 49^{\mathrm{m}} 25^{\mathrm{s}}+16^{\circ} 15^{\prime} \cdot 4(1855) \cdot 217$. E. Schönfeld. Ueber eine Beobachtung des Planeten (29) Amphitrite aus dem Jahre 1847 , nebst Bemerkungen uber eine muthmassliche Beobachtung des Planeten (192) Nausikaa aus demselben Jahre. 2I9. - Eugen v. Gothard. Photographische Aufnahmen. 221. - Todes-Anzeige. 223. Entdeckung eines neuen Cometen von Finlay. 223. - Vermischte Nachrichten. 223. - Verkaufs-Anzeige. 223. 